\title{
Aprovechamiento energético de la biomasa del sorgo y valorización de subproductos
}

\section{Energy use of sorghum biomass and recovery of by-products}

\section{Doctorando:}

\section{Carlos E. PONCIO}

Centro de Investigación y Tecnología Química (CITeQ), Universidad Tecnológica Nacional (UTN), Consejo Nacional de Investigaciones Científicas y Técnicas de Argentina (CONICET) - Argentina

cponcio@frc.utn.edu.ar

\section{Director/es:}

Horacio FALCON

\section{Co-director/es:}

\section{María Soledad RENZINI José Antonio ALONSO}

\begin{abstract}
Resumen
En el presente trabajo se exponen los principales avances obtenidos hasta el momento de la presentación a estas Jornadas Doctorales, considerando que he comenzado mi doctorado en noviembre de 2019. El objetivo de la tesis es estudiar la pirólisis catalítica de la biomasa proveniente del proceso de industrialización del sorgo, usando como catalizadores compuestos de la familia de los pirocloros en especial los de estructura tipo $\left(\mathrm{H}_{3} \mathrm{O}\right) \mathrm{SbTeO}_{6}$, como así también las zeolitas tipo Y y, ZSM-11. El propósito fundamental es obtener altos rendimientos en la fracción líquida, ya sea para ser empleado como potencial combustible o como fuente de otros compuestos de interés. Todo este proceso se llevará a cabo en un horno pirolítico horizontal, empleando temperaturas en un rango comprendido entre los $300^{\circ} \mathrm{C}$ y los $450^{\circ} \mathrm{C}$ durante 20 minutos.

Asimismo, se analizarán los productos gaseosos no condensables para su utilización como otra fuente de energía y del mismo modo se verá la factibilidad de usar al bio-carbón residual como mejorador de suelo o agente filtrante.
\end{abstract}

Palabras clave: Biomasa, Pirólisis, Pirocloros, Catálisis, Biocombustibles.

\begin{abstract}
The purpose of this thesis is to analyze the catalytic pyrolysis of biomass from the industrialization of sorghum, using as catalyst compounds of the pyrochloro family, especially those with the $\left(\mathrm{H}_{3} \mathrm{O}\right) \mathrm{SbTeO}_{6}$ type structure, as well as the Y ultra-stable type zeolites and ZSM-11. The mayor aim of this project is to obtain high yields in the liquid fraction, which can be used as a bio-oil or a source of other compounds of interest. All this process will be carried out in a horizontal pyrolytic oven, testing at a constant temperature in a range between $300{ }^{\circ} \mathrm{C}$ and $450{ }^{\circ} \mathrm{C}$ for 20 minutes.

Likewise, non-condensable gaseous products will be analyzed for their use as another source of energy and in the same way the feasibility of using residual biochar as a soil improver or filter agent will be seen.
\end{abstract}

Keywords: Biomass, Pyrolysis, Pyrchloros, Catalysis, Biofuels. 


\section{Introducción}

En la actualidad tenemos varios problemas acuciantes a nivel mundial y regional, entre ellos el procesamiento de residuos y el cambio en la matriz energética para ir minimizando el uso de los combustibles fósiles. En este escenario surge la Química Verde o Química Sustentable, como búsqueda de alternativas que prevengan la contaminación, sin dejar de producir los materiales necesarios para mantener la calidad de vida de los seres humanos.

Atento a esto, hay un gran cambio de paradigma que se viene dando a todo nivel en lo referente a la apreciación de los residuos como tal, hoy se los considera como materia prima para la generación de compuestos de alto valor agregado o simplemente como fuente de combustible. Es por eso por lo que la biomasa proveniente de residuos de la agroindustria es una valorable fuente de materia prima para los procesos como los que se quiere estudiar en esta tesis.

Al hablar desde el punto de vista químico, la pirólisis es un proceso complejo. Generalmente, se lleva a cabo a través de una serie de reacciones en las que influyen muchos factores: la estructura y composición de la materia prima, la tecnología utilizada, la velocidad de calentamiento, el tiempo de residencia, la velocidad de enfriamiento y la temperatura del proceso.

La propuesta de esta tesis es estudiar la pirólisis de la biomasa procedente del procesamiento de las semillas de sorgo con la finalidad de obtener una fracción sólida (denominada bio-carbón), otra fracción líquida (denominada bio-aceite) y finalmente otra gaseosa (denominada gas pobre). Para lo cual se analizará el beneficio de la utilización de catalizadores ácidos termoestables del tipo pirocloro $\left(\mathrm{H}_{3} \mathrm{O}\right)_{\mathrm{x}} \mathrm{Sb}_{\mathrm{x}} \mathrm{Te}_{(2-\mathrm{x})} \mathrm{O}_{6}(\mathrm{x}=1,1.1$ and 1.25) .

\section{Desarrollo}

En esta primera parte se ha trabajado en diseño y síntesis de los catalizadores sólidos ácidos termoestables para su utilización en la pirólisis del sorgo con la finalidad de la obtención de productos líquidos, sólidos y gaseosos que se utilicen como fuente renovable de energía o de productos de alto valor agregado.

\section{Síntesis de Pirocloros}

Estos materiales se sintetizan mediante un procedimiento de reacción en estado sólido para obtener en primer lugar el pirocloro de potasio (KSbTe06) para luego al ser tratado con ácido sulfúrico concentrado intercambiar los $\mathrm{K}^{+}$por $\mathrm{H}_{3} \mathrm{O}^{+}$.

Una de las ideas es tratar de salir de la relación 1:1 entre el Sb y Te; además de sintetizar otras series como $\mathrm{H}_{3} \mathrm{O}\left(\mathrm{NbTe}_{6} \mathrm{O}_{6}, \mathrm{H}_{3} \mathrm{O}\left(\mathrm{SbMo}_{6} \mathrm{O}_{6}\right.\right.$ y $\mathrm{H}_{3} \mathrm{O}\left(\mathrm{NbMo}_{6} \mathrm{O}_{6}\right.$.

Estas especies sólidas serán estudiadas aplicando las técnicas de: FTIR; SEM; TPD-NH3; BET entre otros.

\section{Caracterización de la biomasa}

Como primera etapa se ha empezado a caracterizar la biomasa que utilizará en la pirólisis, para lo cual se ha empezado por efectuarle un análisis termogravimétrico en una balanza TGA / SDTA851 e /SF $/ 1100^{\circ} \mathrm{C}$ Mettler Toledo. Donde se estudió el comportamiento en atmósfera inerte desde temperatura ambiente hasta los $700{ }^{\circ} \mathrm{C}$, registrándose la pérdida de peso y velocidad de calentamiento durante todo el experimento.

\section{Pirólisis rápida}

Para esta etapa se ha puesto a punto el sistema pirolítico, el cual consiste en un reactor de cuarzo del tipo horizontal, dispuesto dentro de un horno el cual se trabajará a temperaturas entre 300 a $450^{\circ} \mathrm{C}$ y con diferentes relaciones alimentación /catalizador (ver Figura 1).El sistema cuenta con las correspondientes trampas de líquidos (se utilizan dos trampas en cascada refrigeradas con nitrógeno líquido) y gases.

Todo el ensayo se realiza a presión subatmosférica (alrededor de $400 \mathrm{mmHg}$ ) y con un flujo de gas inerte (nitrógeno) de15 mL/min.

La biomasa junto con el catalizador a estudiar se introducirá dentro del reactor de cuarzo en una cápsula de alumina, una vez que el horno se haya estabilizado a la temperatura deseada. Después de los 20 min, se apaga el reactor y se espera que se enfríe, se sigue suministrando flujo gaseoso y se extraerá la trampa de líquido del 
tipo U que se encuentra a la salida del reactor. Una vez alcanzada la temperatura ambiente se procederá a extraer el bio-carbón obtenido con el catalizador utilizado.

La trampa de líquido se lavará con acetona para recolectar toda la masa que pudiere haber quedado impregnando las paredes de esta. Posteriormente se concentrará utilizando un evaporador rotatorio.

Los productos líquidos serán analizados mediante cromatografía de gases en un equipo Varian 3800 acoplado con un detector de espectrómetro de masas con una columna capilar HP-5 de 30m x 0,250 mm Agilent. La caracterización de los productos se realizará por comparación con la biblioteca NIST considerando un match superior a $80 \%$.; mientras que los gaseosos por medio de un GC-TCD.

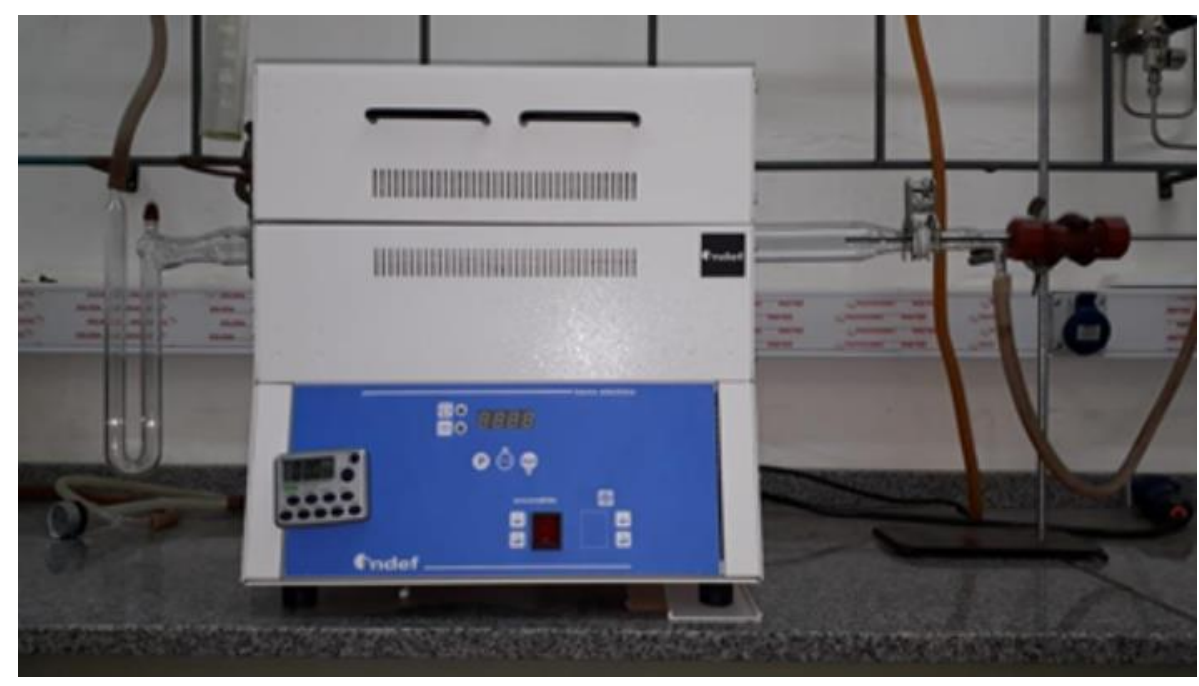

Figura 1. Fotografía del equipo de pirólisis rápida

\section{Resultados}

En esta primera etapa se analizó la biomasa (sorgo) en estudio; la que se encuentra formada principalmente por tres componentes: celulosa, hemicelulosa y lignina. En una primera aproximación, se pueden considerar a los materiales lignocelulósicos como una mezcla entre un 40-80 \%p/p de celulosa, 15-30 \%p/p de hemicelulosa y 10-25\%p/p de lignina. La diferencia en su estructura química conduce a diferentes reactividades, haciendo que la composición de la biomasa sea un factor crucial para el diseño de un proceso. El TGA se utilizó para determinar la cantidad de lignina, hemicelulosa y celulosa en dos clases diferentes de biomasa: sorgo blanco y sorgo marrón (Figuras 2 a y 2 b).
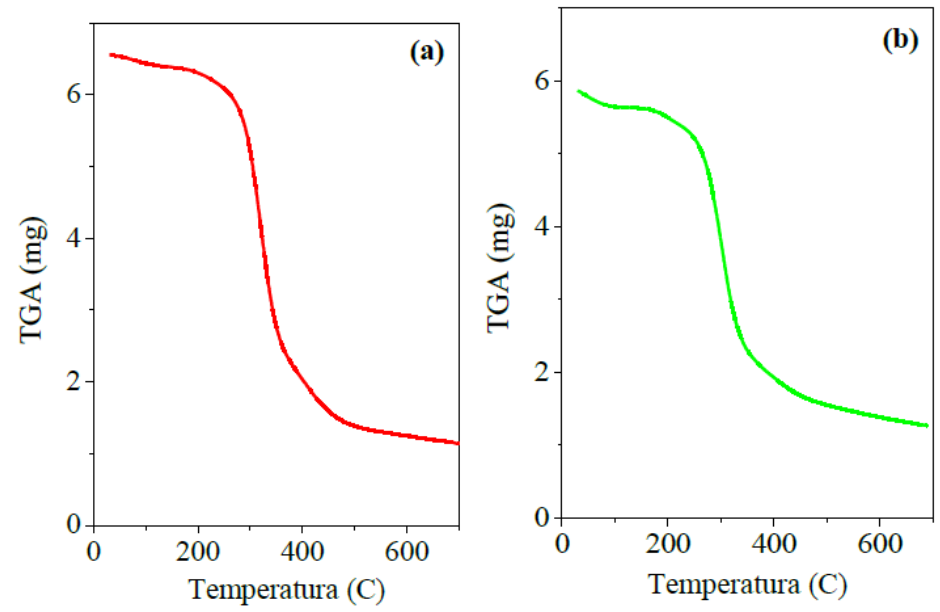

Figura 2. Curvas de TGA: (a) Sorgo blanco y (b) Sorgo marrón. 
Estas curvas muestran que el proceso de desvolatilización comienza a los $150{ }^{\circ} \mathrm{C}$ y la pérdida de peso máxima ocurre en el rango de los $200-350{ }^{\circ} \mathrm{C}$. Pasados los $350^{\circ} \mathrm{C}$ se puede apreciar el cambio brusco de la curva haciendo la pérdida de peso más lenta en el rango $350-400^{\circ} \mathrm{C}$. Se puede observar que entre los $120^{\circ} \mathrm{C}$ y los 500 ${ }^{\circ} \mathrm{C}$ se ha perdido más del $70 \%$ en peso de la materia volátil. En la Figura 3 se aprecian las curvas diferenciales termogravimétricas (DTGA) obtenidas para ambas biomasas.
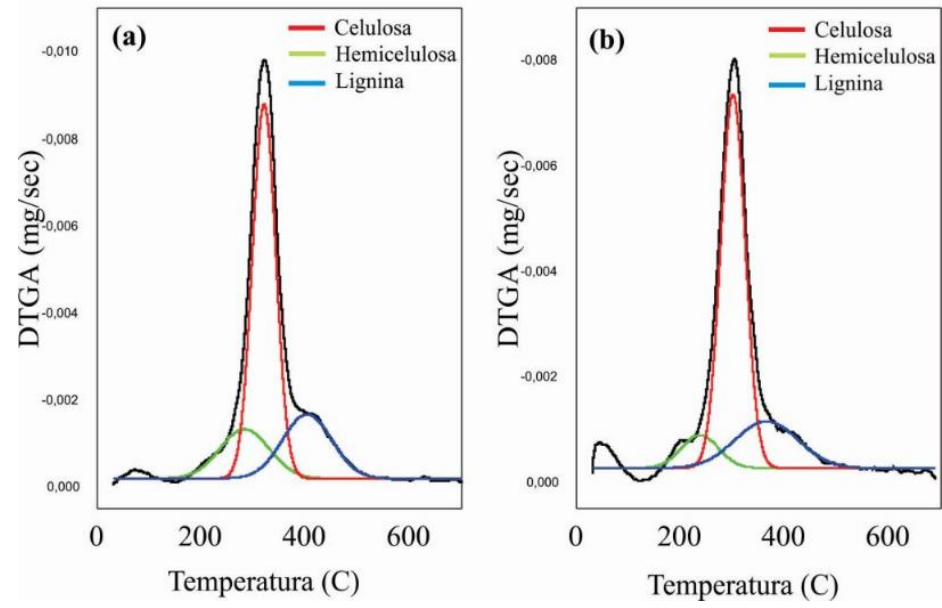

Figura 3. Curvas DTGA con el cálculo de deconvolución: (a) Sorgo blanco y (b) Sorgo marrón.

Esta deconvolución permite la identificación de los principales componentes de la biomasa: celulosa, hemicelulosa y lignina. Las curvas presentan tres picos principales de la degradación de hemicelulosa en el rango de $200-300{ }^{\circ} \mathrm{C}$, la celulosa entre los $250-350{ }^{\circ} \mathrm{C}$, mientras la lignina entre los $350-500{ }^{\circ} \mathrm{C}$. Las intensidades relativas de los picos están relacionadas con las cantidades de cada uno de los componentes presentes en la biomasa.

\section{Determinación estructural del catalizador}

La muestra $\left(\mathrm{H}_{3} \mathrm{O}\right) \mathrm{SbTeO}_{6}$, obtenido mediante intercambio iónico de $\mathrm{KSbTe06}$, así como éste precursor, exhibieron una excelente cristalinidad, como lo evidencia la anchura de picos de las reflexiones de XRD (Figuras 4(a) y 4(b)). El patrón de XRD se indexó con una celda cúbica con a $=10.1510$ Å, característico de una estructura de pirocloro.
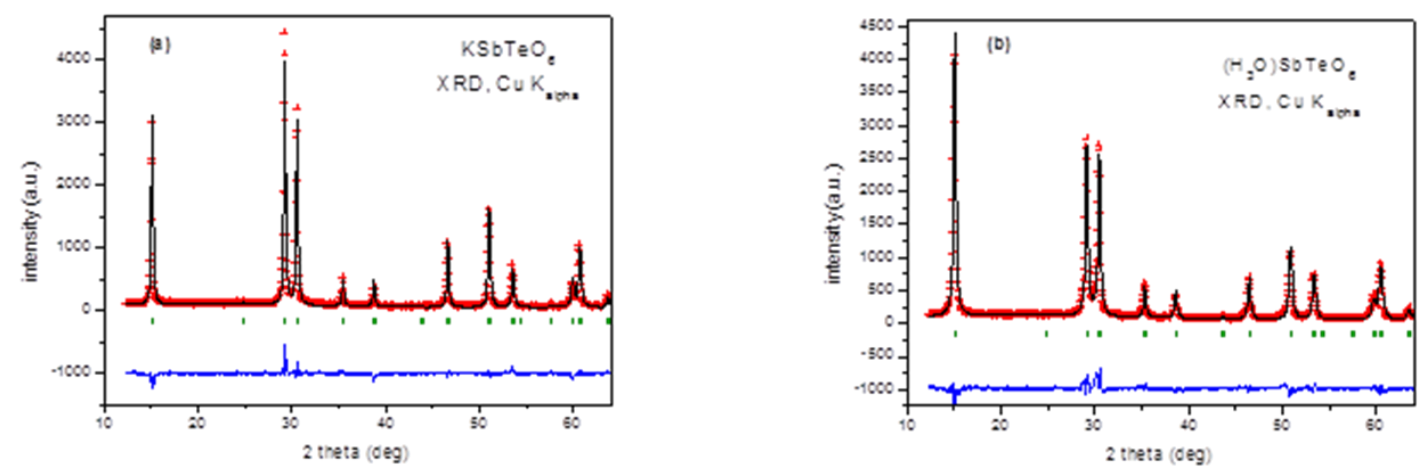

Figura 4. Diagramas de XRD del precursor $\mathrm{KSbTeO}_{6}$ (a) y después del cambio iónico de $\mathrm{K}^{+}$por $\mathrm{H}_{3} \mathrm{O}^{+}$(b), refinados por el método Rietveld 


\section{Conclusiones}

Se ha comenzado a trabajar en la puesta a punto del sistema de pirólisis, así como en la síntesis y caracterización de los catalizadores y de la biomasa de partida. En la etapa siguiente luego de terminar de analizar las variables operativas del sistema de reacción de pirólisis puramente térmica se realizarán los ensayos empleando catalizadores del tipo pirocloros en vista de mejorar la calidad de los productos obtenidos.

\section{Referencias}

Gielen, D., Boshell, F., Saygin, D., Bazilian, M., Wagner, N., Gorini, R. (2019). The role of renewable energy in the global energy transformation. Energy Strategy Reviews, 24, 38-50. doi.org/10.1016/j.esr.2019.01.006

Hofer, R., Bigorra, J. (2008). Biomass-based green chemistry: sustainable solutions for modern economie. Green Chemistry Letters and Reviews, 1, 2, 79-97. doi.org/10.1080/17518250802342519.

Asveld. L. (2019). Towards including social sustainability in green and sustainable chemistry. Current Opinion in Green and Sustainable Chemistry, 19, 61-65. doi.org/10.1016/j.cogsc.2019.06.001

Clark, J.H. (2017). From waste to wealth using green chemistry: The way to long term stability. Current Opinion in Green and Sustainable Chemistry, 8, 10-13. doi.org/10.1016/j.cogsc.2017.07.008

Kohli, K., Prajapati, R., Sharma, B.K. (2019). Bio-Based Chemicals from Renewable Biomass for Integrated Biorefinerie. Energies, 12, 233-273. doi.org/10.3390/en12020233.

Akubo, K., Anas Nahil, M., Williams, P.T. (2019). Pyrolysis-catalytic steam reforming of agricultural biomass wastes and biomass components for production of hydrogen/syngas. Journal of the Energy Institute, 92, 19871996. doi.org/10.1016/j.joei.2018.10.013.

Zabanioutou, A., Ioannidou, O., Antonakou, E., Lappas, L. (2008). Experimental study of pyrolysis for potential energy, hydrogen, and carbon material production from lignocellulosic biomass. International Journal of Hydrogen Energy, 33, 2433-2444. doi.org/10.1016/j.ijhydene.2008.02.080.

Thangalazhy-Gopakumar, S., Adhikari, S., Ravindran, H., Gupta, R.B., Fasina, O. (2010). Physiochemical properties of bio-oil produced at various temperatures from pine wood using an auger reactor. Bioresource Technology, 101, 8389-8395. doi.org/10.1016/j.biortech.2010.05.040.

Iman, T. Capareda, S. (2012). Characterization of bio-oil, syn-gas and bio-char from switchgrass pyrolysis at various temperatures. Journal of Analytical and Applied Pyrolysis 93, 170-177.

doi.org/10.1016/j.jaap.2011.11.010 